\title{
Procesos y Prácticas de Gestión del Conocimiento en Cadenas Productivas de Colombia
}

\author{
Marcelo López ${ }^{(1)}$, Albeiro Hernández ${ }^{(2)}$ y Carlos E Marulanda ${ }^{(3)}$ \\ (1) Universidad de Caldas, Facultad de Ingeniería, Departamento de Sistemas e Informática, \\ Calle 65 No.26-10, Manizales-Colombia. (e-mail: mlopez@ucaldas.edu.co) \\ (2) Universidad Cooperativa Colombia sede Pereira, Centro de investigaciones. La Julita, \\ Pereira-Colombia. (e-mail: albeiro.hernandez@ucc.edu.co) \\ (3) Universidad Nacional de Colombia, Facultad de Administración, Departamento de Administración. \\ Campus Palogrande. Calle 27 No. 64-60, Manizales-Colombia y Universidad de Caldas, Facultad de \\ Ingeniería, Departamento de Sistemas e Informática, Calle 65 No. 26-10, Manizales-Colombia. \\ (e-mail: cemarulandae@unal.edu.co, carlose@ucaldas,edu.co)
}

Recibido Oct. 30, 2013; Aceptado Dic. 4, 2013; Versión final recibida Dic. 17, 2013

\begin{abstract}
Resumen
El objetivo del trabajo que se presenta fue determinar la capacidad de las empresas colombianas para gestionar su conocimiento en algunas de las más importantes cadenas productivas del país. Se evaluaron 321 empresas en relación a las competencias, los procesos y las prácticas para gestionar conocimiento. Se evidencian avances en las cadenas de metalurgia y tecnologías de información y comunicaciones, pero deficiencias en las cadenas de salud y agroindustria. Este estudio aporta a la comunidad académica y a las cadenas productivas del país los elementos necesarios para establecer una línea base del estado de la gestión del conocimiento, que permita plantear acciones alternativas, programas y proyectos para su beneficio.
\end{abstract}

Palabras clave: gestión de conocimiento, evaluación de la gestión del conocimiento, prácticas, procesos, competencias.

\section{Processes and Knowledge Management Practices in Production Chains of Colombia}

\begin{abstract}
The aim of the work was to determine the ability of Colombian companies to manage their knowledge in some of the most important productive chains of the country. A total of 321 companies were evaluated in relation to skills, processes and practices to manage knowledge. Companies show progress in the fields of metallurgy and information and communications technologies, but deficiencies in the health chain and agroindustry. This study contribute to the academic community and the productive chains in the country with the necessary elements for establishing a base of the present situation of knowledge management, that allows proposing alternative actions, programs and projects for their own benefit.
\end{abstract}

Keywords: knowledge management, evaluation of knowledge management practices, processes, competencies. 


\section{INTRODUCCIÓN}

Este trabajo muestra los resultados de la investigación sobre la gestión del conocimiento (GC) en empresas de las principales cadenas productivas de Colombia. Se determina, de qué manera las competencias, prácticas y procesos, como componentes de un modelo de valoración integral de la gestión del conocimiento empresarial, son a su vez, elementos dinamizadores de la gestión de las cadenas productivas. La sociedad actual se caracteriza por la incertidumbre, los mercados desregulados, y la formación de una economía interconectada en tiempo real, el uso de las tecnologías de información y comunicaciones (TI), el desarrollo de la telefonía y computación móvil, los servicios orientados a clientes, la mercadotecnia y la innovación (Alonso, 2006).

De ésta manera, innovar no solo significa desarrollar nuevos productos y transformar los productos existentes, sino que consiste en crear nuevas formas de organizar, gestionar o producir; logrando, en última instancia, generar valor agregado a través de toda la cadena productiva. Es por esto que la innovación se caracteriza por una mayor flexibilidad para responder a las demandas del mercado y por el aumento de las relaciones de cooperación y competencia, lo cual plantea la necesidad de transformar las estrategias de los diversos sectores de la economía (McCormick, 2005). La innovación en el marco de la gestión del conocimiento es un agente de cambio que dinamiza externalidades en los sistemas productivos (Nelson, 1995), (Plaza y González, 2004), convirtiéndose en factor fundamental de la competitividad.

Las razones de uso de la gestión del conocimiento son variados, partiendo de generar cambios y resultados sustentables, pasando por optimizar recursos, aprovechar el conocimiento existente, aprender permanentemente, estimular la creatividad e innovación; lo planteado por (Arthur y Huntley, 2005), (Collins y Smith, 2006), (Mesmer y DeChurch, 2009) y (Lin, 2007), que en sus trabajos investigativos muestran que la combinación e intercambio de conocimientos en una organización se relaciona directamente con: la disminución de costos, la creatividad e innovación de productos, el mejoramiento organizacional, el aumento de su rendimiento y de los ingresos por ventas. La gran mayoría de los estudios en la literatura de gestión del conocimiento sugieren que la gestión del conocimiento tiene un impacto positivo y de mejoramiento del rendimiento de las organizaciones (Nonaka y Takeuchi, 1995), (Yang, 2010), (Zheng et al., 2010).

La gestión del conocimiento se presenta como una disciplina cuyo objetivo se centra en desarrollar el conocimiento en las fases siguientes: adquisición, almacenamiento, transformación, distribución y utilización, con la finalidad de lograr ventajas competitivas (Riesco, 2006) (Barney, 1991), (Dosi, Teece y Winter 1992). Y permite abordar la complejidad del análisis organizacional y de sus interacciones a través de una forma de pensamiento basada en la totalidad y sus propiedades, (Harrison, et. al.,2007), (Oltra, 2012) y (Morin, 1994). El conocimiento como recurso estratégico clave es reivindicado como principal fuente de ventaja competitiva y artífice del logro de rentas superiores (Donate, 2007), (Defries y Malone, 2007). Ahora bien, tal como lo plantea (Chen y Liang, 2011), con el rápido desarrollo del comercio electrónico y la tendencia hacia la globalización, el entorno de las organizaciones está cambiando. Bajo estas circunstancias, los activos de conocimiento se han convertido en una importante fuente de ventajas competitivas para la mayoría de las organizaciones. El conocimiento puede ser evaluado (Andone y Cuza, 2009), para lo cual se adoptan varios enfoques: medir el impacto de la gestión del conocimiento en el desempeño corporativo, el cuadro de mando integral, el retorno de la Inversión (ROI), el ciclo de vida de gestión del conocimiento y las encuestas a empleados.

Según (Tunc, Beskese y Kahraman, 2007), la medición del conocimiento podrá hacerse considerando el capital intelectual abordando tres componentes: el capital relacional, el capital humano y el capital estructural. Estos componentes del capital intelectual los define (Sánchez, 2003), así: i) El capital humano, reside en los miembros de la organización y permite generar valor para la empresa. Este crece de dos maneras, cuando la organización usa más conocimientos de los que posee su gente y cuando esta adquiere más conocimientos para la organización; II) El capital estructural, descrito como aquel conocimiento que la empresa ha podido internalizar y que permanece en la organización, ya sea en su estructura, en sus procesos o en su cultura, aun cuando los empleados abandonan ésta. Por tanto, en esta dimensión se incluyen todos los intangibles que no residen en los miembros de la organización, es decir, desde la cultura y los procesos internos, hasta los sistemas de información y las bases de datos; y iii) El capital relacional, se sustenta en la consideración de que las empresas se relacionan con el exterior, en un primer acercamiento con clientes, proveedores, accionistas y otros grupos de interés que le aportan valor a la organización. Como activo intangible es de vital importancia para la unidad de información, y se debe medir y gestionar la red de relaciones con usuarios, proveedores e instituciones afines.

Para complementar, de acuerdo con (Malhotra, 2003), hay cuatro métodos básicos para la clasificación de modelos de medición del capital intelectual: mercado de capitalización, que muestra la diferencia entre la capitalización de mercado y el capital contable; el método de activos, que se basa en que los activos 
tangibles y las cifras anuales se comparan con la media del sector y por encima de la media las ganancias se utilizan para estimar el valor de los activos intangibles; el método directo del capital intelectual, en el cual los componentes son identificados y valorados; y el método Scorecard, en el cual los componentes del capital intelectual se identifican y se reflejan en un cuadro de mando.

Igualmente, (Sánchez, 2003), explica que existen varios modelos para la gestión del capital intelectual. Algunos de los modelos más conocidos son modelo de Sullivan, el Capital Intelectual de Skandia, el modelo Brooking, el modelo de categorización de Roos, el modelo de Onge, el modelo de Sveiby y el modelo de Wiig. Se tienen presentes los resultados de investigaciones a nivel internacional sobre: los servicios intensivos en conocimientos de negocios (Strambach, 2008), (Feldkamp, Hinkelmann y Thönssen, 2007), la construcción de los fundamentos teóricos de economía de la información (Salas, 2004), (Andriessen, 2008), la gestión estratégica (Kong y Thomson, 2009), la cultura organizacional (Smith, McKeen y Singh, 2011), el comportamiento organizacional, estructura organizativa, inteligencia artificial, la calidad (Tarí y García, 2009), la gestión y medición del desempeño organizacional (Yang y Wang, 2011), y la analogía conceptual entre un ecosistema de innovación y los ecosistemas biológicos observados en la naturaleza, entre otros.

En Colombia se atienden los resultados de investigaciones en torno a: el grado de coincidencia entre el concepto y el enfoque que tienen las organizaciones sobre la gestión del conocimiento (Briceño y Bernal, 2010), la valoración de la sociedad del conocimiento (Marulanda, Giraldo y López, 2013), el capital intelectual y el desarrollo regional (Hernandez y Lasso, 2011), la identificación del aprendizaje organizacional y la generación de capital intelectual en la gestión del conocimiento (Hincapie, 2009), la generación de valor y el fortalecimiento de las ventajas competitivas de las empresas (Marín y Atehortúa, 2010), el modelo de industrialización acorde con las necesidades de las regiones colombianas (Joyanes, Cuesta y López, 2010)

Para el caso de este trabajo, se evaluaron las capacidades de las empresas para desarrollar una efectiva gestión del conocimiento tomando algunos de los sectores económicos definidos por el Banco de la República de Colombia (Banco de la República, 2005) y asociados a las cadenas productivas, como son: agroindustria, metalmecánica, alimentos, comercio, salud, construcción, servicios, informática y software. Se presentan los resultados de la aplicación de un modelo de valoración de la gestión del conocimiento para empresas, mediante una evaluación integral y permanente de las siguientes categorías: competencias, prácticas y procesos, en 321 PYMES de ciudades colombianas: Armenia, Barranquilla, Bogotá, Bucaramanga, Cali, Medellín, Manizales y Pereira.

\section{MATERIALES Y MÉTODOS}

Para el desarrollo se hace una investigación exploratoria, para analizar e identificar las principales variables del modelo de evaluación, una investigación descriptiva, para conocer las situaciones predominantes con la representación exacta de los procesos, y una investigación de tipo correlacional para encontrar la relación entre las variables.

\section{Determinación de la muestra}

Se aplicó la encuesta a través de internet a una muestra de la población de 321 PYMES de diversas regiones de Colombia. Previamente a la valoración, se desarrolló una prueba piloto de la encuesta con expertos sobre el tema, además de una revisión con algunos de los actores académicos y del ámbito productivo. Para la estructuración del cuestionario se formularon preguntas en escala Likert, las cuales se calificaron de 1 a 5, donde 1 no realizado, 2 realizado parcialmente, 3 realizado en intervalos de tiempo, 4 realizado con regularidad y 5 realizado completamente.

\section{Categorías y variables evaluadas}

Las categorías y variables se fundamentan en los modelos y planteamientos desde la perspectiva europea, (CEN, 2004), el foro europeo de conocimiento, (Chen, 2008), y los desarrollos de autores como (Bueno, 2003), y (López, 2010), en los que se define la medición desde el capital humano, organizativo, tecnológico, relacional e intangible, entre otros, considerando las interrelaciones y las perspectivas dinámicas de una organización del conocimiento, por medio de métricas, herramientas e indicadores específicos, ligados a las competencias individuales y las capacidades organizacionales. Estas se agruparon tal como se observa en la tabla 1. Para revisión de la herramienta de encuesta y los resultados, ingresar al portal www.logopoliskpo.com/sigeco, con el usuario: carloselog@gmail.com y la contraseña: carlose1999. 
Tabla 1. Categorías y variables a evaluar

\begin{tabular}{|l|l|c|}
\hline \multicolumn{1}{|c|}{ Categorías } & \multicolumn{1}{|c|}{ Variables } & № de preguntas \\
\hline $\begin{array}{l}\text { Competencias (personales } \\
\text { para la gestión del } \\
\text { conocimiento) }\end{array}$ & $\begin{array}{l}\text { Gestión de la información y la documentación, gestión de la } \\
\text { comunicación, diseño de herramientas digitales, gestión de la } \\
\text { innovación y el cambio y gestión del aprendizaje organizacional }\end{array}$ & 44 \\
\hline $\begin{array}{l}\text { Prácticas (herramientas } \\
\text { para la gestión del } \\
\text { conocimiento) }\end{array}$ & Identificar, generar, retener, compartir y aplicar conocimiento & 33 \\
\hline $\begin{array}{l}\text { Procesos (fases del ciclo } \\
\text { de vida de la gestión del } \\
\text { conocimiento) }\end{array}$ & $\begin{array}{l}\text { Aspectos (estrategias), procesos para identificar, generar, retener, } \\
\text { compartir y aplicar conocimiento }\end{array}$ & 52 \\
\hline
\end{tabular}

\section{RESULTADOS Y DISCUSIÓN}

El proceso de recolección de datos se hizo personal y telefónicamente con representantes de las empresas en el momento del registro, para asegurarse que tuvieran comprensión de procesos relacionados con la gestión del conocimiento organizacional, además de hacer un muestreo aleatorio de comprobación. Se presenta, a su vez, la matriz de correlación, ver tabla 2, la cual enseña un alto grado de correlación entre las categorías evaluadas, lo que hace posible aplicar el método de componentes principales.

Tabla 2.Tabla de correlaciones (valor $p$ )

\begin{tabular}{|c|c|c|c|c|c|c|c|c|c|c|c|c|c|c|c|c|}
\hline Variables & 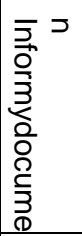 & 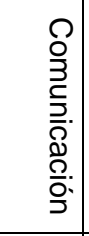 & 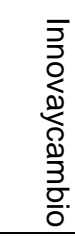 & 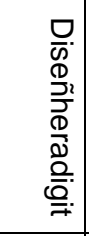 & $\begin{array}{l}\frac{D}{0} \\
\frac{0}{0} \\
\frac{0}{0} \\
\frac{0}{N} \\
0 \\
0 \\
0 \\
0\end{array}$ & $\begin{array}{l}\overline{\mathrm{O}} \\
\bar{D} \\
\overline{\bar{B}} \\
\bar{D} \\
\overline{0} \\
\overline{0}\end{array}$ & 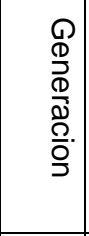 & 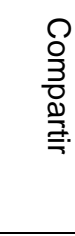 & 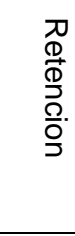 & $\begin{array}{l}\frac{D}{2} \\
\text { 음. } \\
\frac{0}{0} \\
\text { ㅇ. }\end{array}$ & $\begin{array}{l}0 \\
D \\
0 \\
0 \\
0 \\
0\end{array}$ & 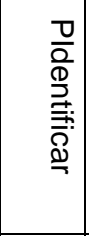 & $\begin{array}{l}\text { D } \\
\mathbb{\Phi} \\
\stackrel{\mathbb{D}}{\mathbb{D}} \\
\stackrel{\mathbb{D}}{\longrightarrow}\end{array}$ & $\begin{array}{l}\text { D } \\
\stackrel{\mathbb{D}}{\mathbb{D}} \\
\stackrel{\mathbb{\Phi}}{\mathbb{D}}\end{array}$ & 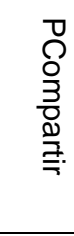 & $\begin{array}{l}\frac{D}{2} \\
\frac{\overline{\bar{D}}}{2} \\
\stackrel{2}{n}\end{array}$ \\
\hline Informydocumen & & ,001 & ,004 & ,048 & ,014 & ,625 & ,304 & ,782 & ,040 & ,834 & ,025 & \begin{tabular}{|l|}
, 044 \\
\end{tabular} & ,293 & ,046 & ,036 & 017 \\
\hline Comunicación & ,001 & & ,000 & ,348 & ,002 & ,048 & 039 & ,329 & ,037 & ,445 & ,050 & ,069 & ,042 & ,943 & ,029 & 016 \\
\hline Innovaycambio & 004 & , 000 & & ,313 & ,003 & ,975 & , 809 & ,510 & ,329 & ,453 & ,016 & , 108 & ,019 & ,749 & ,034 &, 021 \\
\hline Diseñherramdigit & 048 & , 348 & ,313 & & 169 & ,039 & ,011 & ,566 & ,023 & ,338 & ,014 & ,667 & ,039 & 892 &, 026 & ,007 \\
\hline Aprendizaorg & ,014 & ,002 & ,003 & , 169 & & ,529 & 847 & ,032 & 698 & ,516 & ,013 & 241 & ,011 & ,559 & ,698 & ,018 \\
\hline Identificacion & ,625 & ,048 & ,975 & ,039 & ,529 & & ,021 & ,010 & 048 & ,415 & 017 & ,372 &, 043 & ,010 & 041 & ,026 \\
\hline Generacion & ,304 & ,039 & ,809 & ,011 & 847 & ,021 & & ,093 & 280 & ,363 & 026 & , 185 & ,021 & ,699 & 011 &, 036 \\
\hline Compartir & ,782 & ,329 &, 510 & ,566 & ,032 & ,010 & ,093 & & ,031 & \begin{tabular}{|l|}
, 845 \\
\end{tabular} & ,046 & ,619 & ,001 &, 048 & ,686 & ,038 \\
\hline Retencion & ,040 & ,037 & ,329 & ,023 & ,698 & ,048 & ,280 & ,031 & & \begin{tabular}{|l|}
, 032 \\
\end{tabular} & ,029 & \begin{tabular}{|l|}
, 836 \\
\end{tabular} & ,044 & ,063 & ,022 & ,023 \\
\hline Aplicación & ,834 & ,445 & ,453 & ,338 & ,516 & ,415 & ,363 & ,845 & ,032 & & ,784 & ,024 & 697 & 029 & ,282 & ,026 \\
\hline PAspgen & ,025 & ,050 &, 016 & ,014 & ,013 & ,017 & ,026 & ,046 &, 029 & ,784 & & ,011 & ,888 & 029 & 017 & 014 \\
\hline Pidentificar & ,044 & ,069 & , 108 & ,667 & ,241 & ,372 & ,185 & 619 & 836 & ,024 & ,011 & & ,932 & ,983 & ,023 & ,027 \\
\hline PReter & ,293 & ,042 & 019 & 039 & ,011 & ,043 & 021 & 001 & 044 & ,697 & 888 & ,932 & & ,695 & 898 & ,783 \\
\hline PGen & ,046 & 943 & ,749 & ,892 & (559 & ,010 & ,699 & ,048 & 063 & , 029 & 029 & -983 & ,695 & & ,058 &, 565 \\
\hline PCompartir & ,036 & ,029 & ,034 & , 026 & 698 & , 041 & ,011 & ,686 & 022 & 282 & ,017 & ,023 & ,898 & 058 & &, 020 \\
\hline PAplicar & ,017 & ,016 & ,021 & ,007 & ,018 & ,026 & ,036 & ,038 & ,023 &, 026 & ,014 & ,027 & 783 & ,565 & , 020 & \\
\hline
\end{tabular}

Convenciones: gestión de la información y la documentación (informydocumen), gestión de la comunicación (comunicación), gestión de la innovación y el cambio (innovaycambio) diseño de herramientas digitales (diseñherramdigit), y gestión del aprendizaje organizacional (aprendizaorg), aspectos (paspgen), procesos para identificar (pidentifica), retener (pretener), generar (pgenerar), compartir (pcompartir) y aplicar (paplicar) conocimiento. En la categoría competencias para la gestión del conocimiento, los resultados se graficaron tal como se observa en la figura 1.

De acuerdo a la escala de calificación, el promedio de las variables evaluadas está alrededor de 3,6, lo que muestra una calificación medio alta en las competencias para gestionar conocimiento en las diversas cadenas productivas del país. Se destacan valores por debajo del promedio en la cadena de servicios de salud, en la innovación y el cambio y la comunicación, lo que refleja los problemas que viene teniendo esta cadena, además de las más bajas calificaciones de los encuestados. Mientras se destacan resultados por encima del promedio en las competencias para gestionar conocimiento de las cadenas productivas de construcción, alimentos y bebidas y TIC, los cuales en estos momentos están jalonando el desarrollo de Colombia. 


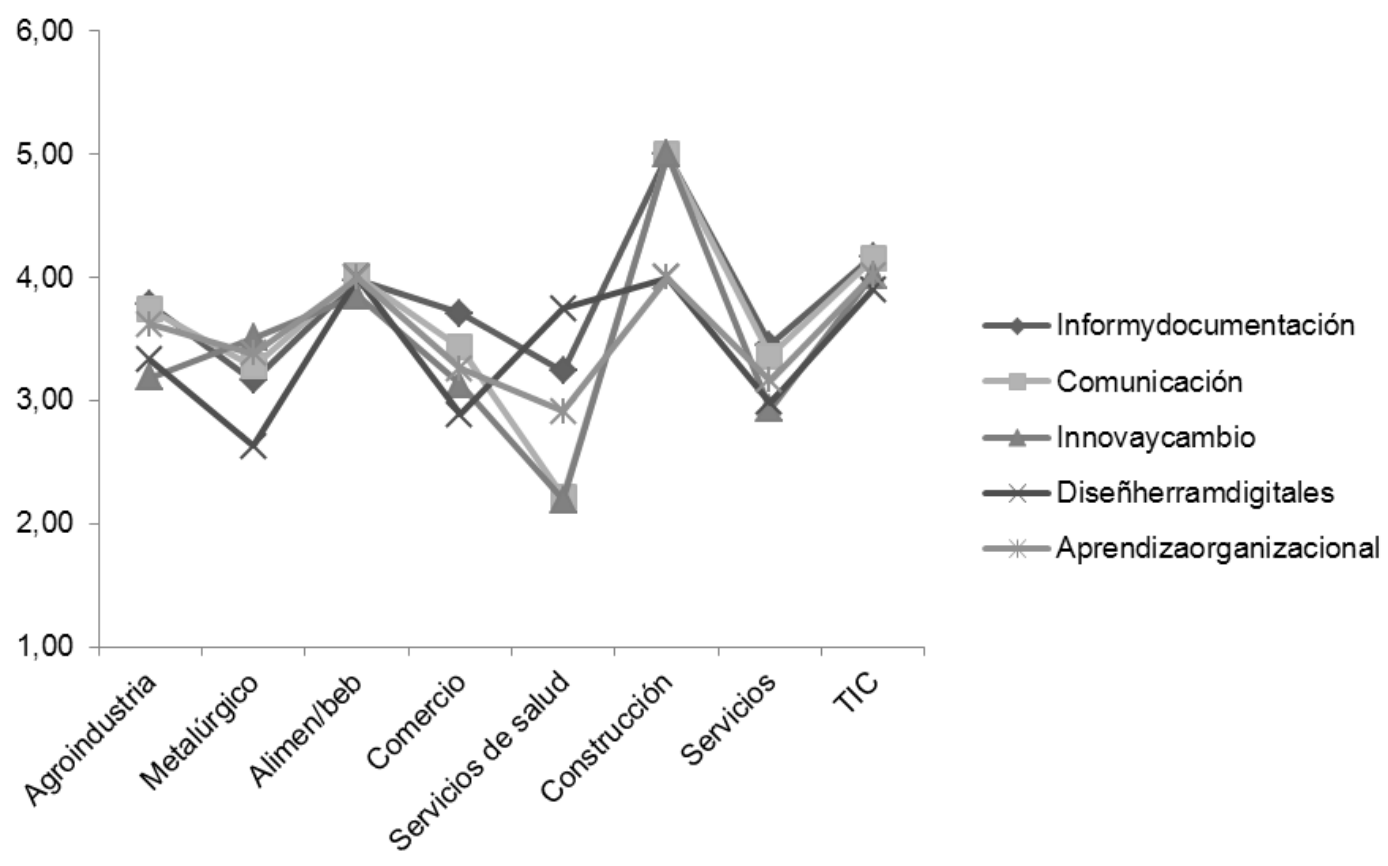

Fig. 1: Competencias para la gestión del conocimiento

En la categoría prácticas para la gestión del conocimiento, los resultados se graficaron tal como se observa en la figura 2.

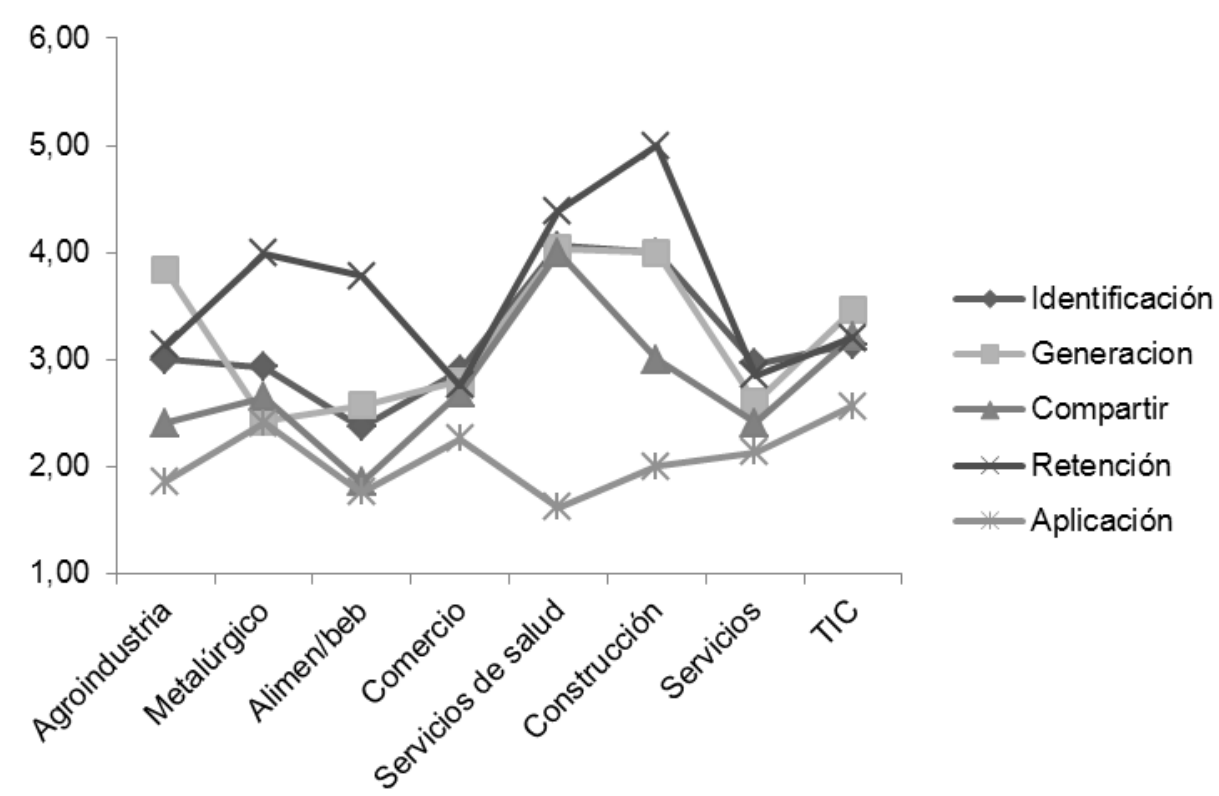

Fig. 2: Prácticas para la gestión del conocimiento

De acuerdo a la escala de calificación, el promedio de las variables evaluadas está alrededor de 3,0. Se destacan valores por debajo del promedio en las cadenas de comercio y servicios, en la identificación, generación, compartición, retención y aplicación de conocimiento. Pero se observa que la variable aplicación es negativa en todas las cadenas productivas. Sobresalen resultados por encima del promedio de las prácticas organizacionales para gestionar conocimiento, en algunas variables, de las cadenas de la construcción y de los servicios de salud. Se observa como la cadena de TIC está en una calificación promedio, debiendo ser uno de los que estableciera diferencia en este campo. En la categoría procesos para la gestión del conocimiento, los resultados se graficaron tal como se observa en la figura 3. 


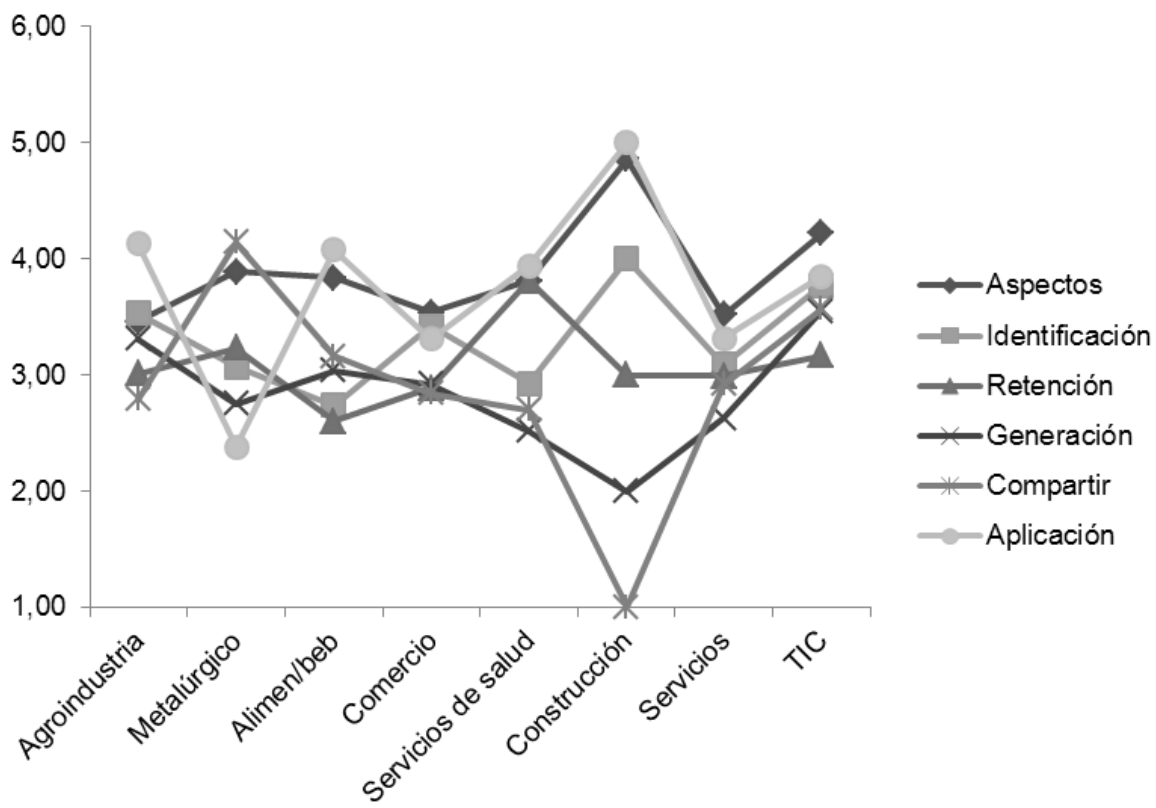

Fig. 3: Procesos para la gestión del conocimiento

De acuerdo a la escala de calificación, el promedio de las variables evaluadas está alrededor de 3,3 . Se presentan valores por debajo del promedio en la cadena de la construcción, desde compartir y generar. Mientras se cuenta con resultados por encima del promedio en el desarrollo de procesos para gestionar conocimiento en las cadenas de servicios y de TIC, en cuanto a identificar, retener, generar, compartir y aplicar conocimiento, así como la identificación y aplicación en la cadena de la construcción.

Dada la cantidad de variables que se evaluaron, se realizó un análisis de componentes principales, con el software estadístico SPSS para determinar, cuáles de ellas explican la gestión del conocimiento en las cadenas productivas del país, ver tabla 3.

Tabla 3.Componentes principales.

\begin{tabular}{|c|c|c|c|c|c|c|}
\hline \multirow[b]{2}{*}{ Componente } & \multicolumn{3}{|c|}{ Autovalores iniciales } & \multicolumn{3}{|c|}{ Sumas de las saturaciones al cuadrado } \\
\hline & Total & $\%$ de varianza & $\%$ acumulado & Total & $\%$ de varianza & $\%$ acumulado \\
\hline 1 & 6,869 & 42,933 & 42,933 & 6,869 & 42,933 & 42,933 \\
\hline 2 & 4,600 & 28,749 & 71,682 & 4,600 & 28,749 & 71,682 \\
\hline 3 & 1,867 & 11,666 & 83,348 & 1,867 & 11,666 & 83,348 \\
\hline 4 & 1,411 & 8,821 & 92,169 & 1,411 & 8,821 & 92,169 \\
\hline 5 & ,998 & 6,237 & 98,406 & & & \\
\hline 6 & ,219 & 1,371 & 99,777 & & & \\
\hline 7 & ,036 & ,223 & 100,000 & & & \\
\hline 8 & $4,252 \mathrm{E}-16$ & $2,658 \mathrm{E}-15$ & 100,000 & & & \\
\hline 9 & $3,150 \mathrm{E}-16$ & $1,969 \mathrm{E}-15$ & 100,000 & & & \\
\hline 10 & $1,929 \mathrm{E}-16$ & $1,205 \mathrm{E}-15$ & 100,000 & & & \\
\hline 11 & $1,357 \mathrm{E}-16$ & $8,479 \mathrm{E}-16$ & 100,000 & & & \\
\hline 12 & $5,771 \mathrm{E}-17$ & $3,607 \mathrm{E}-16$ & 100,000 & & & \\
\hline 13 & $-4,204 \mathrm{E}-17$ & $-2,628 \mathrm{E}-16$ & 100,000 & & & \\
\hline 14 & $-1,536 \mathrm{E}-16$ & $-9,602 E-16$ & 100,000 & & & \\
\hline 15 & $-2,103 E-16$ & $-1,314 \mathrm{E}-15$ & 100,000 & & & \\
\hline 16 & $-2,547 E-16$ & $-1,592 \mathrm{E}-15$ & 100,000 & & & \\
\hline
\end{tabular}

Con base en estos resultados (leer porcentaje acumulado de las líneas 1, 2, 3 y 4) se establece lo siguiente: el primer componente explica el $43 \%$ de los resultados, el segundo explica el $71,6 \%$, el tercero explica el $83,3 \%$ y el cuarto explica el $92,1 \%$. Se procede entonces a asignar a cada variable evaluada un componente, de acuerdo al número mayor y como se observa en la tabla 4 . El resultado de dicha asignación se observa en la tabla 5. 
Tabla 4.Matriz de componentes.

\begin{tabular}{|l|r|r|r|r|}
\hline & \multicolumn{4}{|c|}{ Componente } \\
\cline { 2 - 5 } & \multicolumn{1}{|c|}{1} & \multicolumn{2}{c|}{2} & \multicolumn{1}{c|}{3} \\
\hline Informydocument &, 926 &,- 346 &, 002 &, 036 \\
\hline Comunicación &, 755 &,- 640 &, 052 &,- 088 \\
\hline Innovaycambio &, 786 &,- 542 &, 085 &,- 225 \\
\hline Diseñherramdigit &, 722 &, 128 &,- 219 &, 494 \\
\hline Aprendizaorganizac &, 654 &,- 685 &, 021 &, 171 \\
\hline Identificacion &, 520 &, 819 &, 185 &,- 109 \\
\hline Generacion &, 640 &, 557 &, 157 &, 427 \\
\hline Compartir &, 175 &, 828 &, 481 &, 081 \\
\hline Retencion &, 626 &, 456 &,- 168 &,- 358 \\
\hline Aplicación &,- 115 &,- 545 &, 791 &,- 205 \\
\hline PAspgen &, 889 &, 037 &, 224 &,- 273 \\
\hline PIdentificar &, 676 &,- 163 &, 596 &, 000 \\
\hline PRetener &,- 107 &, 882 &, 409 &, 070 \\
\hline PGenerar &,- 381 &,- 479 &, 356 &, 684 \\
\hline PCompartir &,- 775 &,- 275 &, 292 &, 135 \\
\hline PAplicar &, 868 &, 098 &,- 282 &, 376 \\
\hline
\end{tabular}

Tabla 5. Asignación.

\begin{tabular}{|l|c|}
\hline \multicolumn{1}{|c|}{ Variable } & $\begin{array}{c}\text { Representada por el } \\
\text { factor de componente }\end{array}$ \\
\hline Informydocument & 1 \\
\hline Comunicación & 1 \\
\hline Innovaycambio & 1 \\
\hline Diseñherramdigit & 1 \\
\hline Aprendizaorganizac & 1 \\
\hline Identificacion & 1 \\
\hline Generacion & 2 \\
\hline Compartir & 1 \\
\hline Retencion & 3 \\
\hline Aplicación & 1 \\
\hline PAspgen & 1 \\
\hline Pldentificar & 2 \\
\hline PRetener & 4 \\
\hline PGenerar & 3 \\
\hline PCompartir & 1 \\
\hline PAplicar & 1 \\
\hline
\end{tabular}

Posteriormente se da nombre a estos factores, así:

Factor 1: Competencias para Gestión Conocimiento.

Factor 2: Compartir Conocimiento.

Factor 3: Aplicar Conocimiento.

Factor 4: Generar Conocimiento.

Con estos factores se hace la matriz de correlaciones para corroborar que efectivamente los componentes están bien agrupados (ver tabla 6). Los resultados son los mostrados en la Tabla 6. Estos muestran un nivel alto de significancia entre los nuevos 4 factores y ratifica que la gestión del conocimiento en las cadenas productivas del país, se está generando desde las competencias, el compartir, el aplicar y el generar conocimiento. 
Tabla 6.Correlaciones entre factores obtenidos.

\begin{tabular}{|l|l|r|r|r|r|}
\hline \multicolumn{2}{|l|}{} & $\begin{array}{c}\text { Puntuación de } \\
\text { factor 1 para } \\
\text { análisis 1 }\end{array}$ & $\begin{array}{c}\text { Puntuación de } \\
\text { factor 2para } \\
\text { análisis 1 }\end{array}$ & $\begin{array}{c}\text { Puntuación de } \\
\text { factor 3para } \\
\text { análisis 1 }\end{array}$ & $\begin{array}{c}\text { Puntuación de } \\
\text { factor 4para } \\
\text { análisis 1 }\end{array}$ \\
\hline $\begin{array}{l}\text { Puntuación de factor } \\
1 \text { para análisis 1 }\end{array}$ & Sig. (bilateral) & 1,000 & 1,000 & 1,000 \\
\hline $\begin{array}{l}\text { Puntuación de factor } \\
\text { 2para análisis 1 }\end{array}$ & Sig. (bilateral) & 1,000 & & 1,000 & 1,000 \\
\hline $\begin{array}{l}\text { Puntuación de factor } \\
\text { 3para análisis 1 }\end{array}$ & Sig. (bilateral) & 1,000 & 1,000 & & 1,000 \\
\hline $\begin{array}{l}\text { Puntuación de factor } \\
\text { 4para análisis 1 }\end{array}$ & Sig. (bilateral) & 1,000 & 1,000 & & 1,000 \\
\hline
\end{tabular}

\section{CONCLUSIONES}

De los resultados encontrados, se pueden obtener las siguientes conclusiones, sobre la gestión del conocimiento de las cadenas productivas de Colombia:

Las cadenas productivas más afectadas por el débil desarrollo de la gestión del conocimiento son la comercial, la de servicios y la de agroindustria, las cuales, dados los avances de la economía del país debieran marcar mucho más progreso, considerando además las posibilidades que ofrecen los recientes tratados de libre comercio firmados por el gobierno nacional.

Se nota un avance importante en las cadenas de TIC y metalurgia, las cuales, vienen reconociendo la importancia de la gestión del conocimiento en su desarrollo, pero como en otras cadenas, tiene mucho por mejorar, en especial con la aplicación y el uso de herramientas digitales. En este campo la cadena TIC debiera ser líder, dada su naturaleza de apropiación y aplicación de diversas prácticas tecnológicas.

Con los resultados de esta investigación, se evidencia la necesidad que tienen las empresas de incorporar la gestión del conocimiento como parte de sus estrategias de innovación y desarrollo, desde la formación de su capital intelectual, el mejoramiento de una cultura organizacional, la generación de inventivos, el uso de tecnologías especializadas, el rediseño de las estructuras organizacionales, hasta la realización de inversiones en tecnología de punta, que permitan afrontar los cambios del mundo actual.

Se recomienda que las empresas adopten prácticas de gestión del conocimiento como: sistemas de lecciones aprendidas, gestión de contenidos empresariales, trabajo en redes especializadas de información y conocimiento, sistemas de vigilancia tecnológica e inteligencia competitiva, páginas blancas, páginas amarillas, herramientas para la solución de problemas, trabajo en equipo, trabajo colaborativo, comunidades de práctica, sistemas de gestión de clientes y gestión de requerimientos.

\section{REFERENCIAS}

Alonso, L.E. y C.J. Fernández, El imaginario Managerial: El discurso de la fluidez en la sociedad económica, Política y Sociedad: 43(2), 127-151 (2006),

Andone, I.I. y A.I. Cuza, Measuring the Performance of Corporate Knowledge Management Systems, Informática Económica: 13(4), 24-31 (2009).

Andriessen, D.G., Stuff or love? How metaphors direct our efforts to manage knowledge in organisations, Research \& Practice: 6, 5-12 (2008).

Arthur, J.B. y C.I. Huntley, Ramping up the organizational learning curve: Assessing the impact of deliberate learning on organizational performance under gainsharing, Academy of Management Journal: 48(6), 1159-1170 (2005).

Barney, J., Firm Resources and sustained competitive advantage, Journal of management: 17(1), 99-120 (1991), http://business.illinois.edu/josephm/BA545_Fall\%202011/S10/Barney\%20\%281991\%29.pdf Acceso: 06 de Agosto (2013).

Briceño, M.A. y C.A. Bernal, Estudios de caso sobre la gestión del conocimiento en cuatro organizaciones Colombianas líderes en penetración de mercado, Estudios gerenciales: 26(117), 173-193 (2010).

Bueno, E., Gestión del Conocimiento: desarrollos teóricos y aplicaciones. 2ª edición, 25-38, Ediciones La Coria, Cáceres, España (2003). 
CEN, Comité Europeo de Normalización, European Guide to good practice in knowledge management - Part 1: Knowledge Management Framework, Bruselas, CEN (2004), ftp://cenftp1.cenorm.be/PUBLIC/CWAs/eEurope/KM/CWA14924-01-2004-Mar.pdf. Acceso: 4 de julio (2012).

Chen, D.N. y T.P. Liang, Knowledge evolution strategies and organizational performance: A strategic fit analysis, Electronic Commerce Research and Applications: 10(1), 75-84 (2011).

Chen, T., A multiple-layer knowledge management system framework considering user knowledge privileges, International Journal of software engineering and knowledge engineering: 19(3), $361-387$ (2008).

Collins, C.J. y K.G. Smith, Knowledge exchange and combination: The role of human resource practices in the performance of high-technology firms, Academy of Management Journal: 49(3), 544-560 (2006).

Defries, R.S. y T.F. Malone, (11 de 11 de 2007).Global Change and our Common Future.Obtenido de Wolrd Comisión on Environment and Development: http://www.nap.edu/catalog/1411.html

Donate, M., Estrategias de conocimiento e innovación, Editorial Consejo económico y social, Madrid, España (2007).

Dosi, G., D., Teece y S. Winter, Toward a theory of corporate coherence: preliminary remarks, En Technology and Enterprise in a Historical Perspective, Oxford : Clarendon Press ; New York: Oxford University Press, USA (1992),

http://trove.nla.gov.au/work/20422348?versionld=24154413 Acceso: 09 de Agosto (2013)

Feldkamp, D., K. Hinkelmann y B. Thönssen, KISS - Knowledge-Intensive Service Support: An approach for agile process management, International Symposium Rule ML Advances in Rule Interchange and Applications, 25 - 38 , Orlando (Florida), USA, 25 y 26 de Octrubre (2007).

Harrison, J.R., Z. Lin, G. Carroll y K. Carley, Simulation modeling in organizational and management research, Academy of Management Review: 32(4), 1229 - 1245 (2007).

Hernández, R.A. y N.C. Lasso, Gestión del conocimiento y capital intelectual en el Area Metropolitana Centro Ocidente de Colombia, Memorias: 9(15), 183-194 (2011).

Hincapié, C.A., Gestión del conocimiento, capital intelectual y comunicación en grupos de investigación, 27, 1-25 (2005), http://revistavirtual.ucn.edu.co/index.php/RevistaUCN/article/view/97/192. Acceso: 3 de Mayo (2011).

Joyanes, L., A. Cuesta y M. López, Caracterización de la industria del software en el triángulo del café, Entre ciencia e ingeniería: 4(7), 76-87 (2010).

Kong, E. y S.B. Thomson, An intelectual capital perspective of human resource strategies and practices,Knowledge Management Research \& Practice: 7, 356-364 (2009).

Lin, H.F., Knowledge sharing and firm innovation capability: An empirical study, International Journal of Manpower: 28(3/4), 315-332 (2007).

López, M., Ciudadanía Digital, un modelo de implantación en la región de Manizales y Caldas, Tesis de doctorado, Departamento de Ingeniería, Universidad Pontificia de Salamanca, Madrid, España (2010).

McCormick, D. El futuro de los clusters y las cadenas productivas. Semestre Económico , 8 (15), 87-102. (2005).

Malhotra,Y., Measuring knowledge assets of a nation: knowledge systems for development (2003).

Marín, L. y L. Atehortúa, Comunidades de práctica, una estrategia para la democratización del conocimiento en las organizaciones, una reflexión, Revista Ingenierías Universidad de Medellín, 9 (16), 141-150. (2010).

Marulanda, C.E., Giraldo, J.A. y M. López, Evaluación de la Gestión del Conocimiento en las Organizaciones de la Red de Tecnologías de Información y Comunicaciones del Eje Cafetero en Colombia, Revista Información Tecnológica: 24(4), 105-116 (2013).

Mesmer, J.R. y L.A. DeChurch, Information sharing and team performance: A meta-analysis, Journal of Applied Psychology: 94(2), 535-546 (2009).

Morin, E., Introducción al Pensamiento Complejo, Gedisa, Barcelona, España (1994).

Nelson, R.R., Recent Evolutionary Theorizing About Economic Change, Journal of Economic Literature: 33, 48-90 (1995).

Nonaka, I. y H.Takeuchi, The Knowledge-Creating Company. University Press. (1995).

Plaza, R. y N. González, La Gestión del Conocimiento Organizativo. Dinámicas de agregación de valor en la organización, Revista Economía Industrial: 35 (7), $41-54$ (2004), 
Oltra C.V., La complejidad del conocimiento: retos para su eficaz creación y transferencia en la organización innovadora. Estudios gerenciales. [online]. 28, 57-80 (2012),

Plaza, R. y N. González, La Gestión del Conocimiento Organizativo. Dinámicas de agregación de valor en la organización, Revista Economía Industrial: 357, 41-54 (2004),

Riesco, M., El negocio es el conocimiento, Ediciones Díaz de Santos, Madrid, España (2006).

Salas, F.V., Economía del diseño organizativo, Revista de Contabilidad, 7, 123-160, (2004).

Sánchez, A., Modelo para la medición del capital intelectual de territorios insulares: una aplicación al caso de Gran Canaria, Tesis de doctorado, Universidad de las Palmas de Gran Canaria, Facultad de Ciencias Económicas y Empresariales, Palmas de Gran Canaria, España (2003), http://www.eumed.net/tesisdoctorales/2006/ajsm/Acceso: 06 de agosto (2013).

Smith, H., J. McKeen y S. Singh, Creating the KM mindset: why is it so difficult?, Knowledge Management Research \& Practice: 8, 112-120 (2011).

Strambach, S., Knowledge Commodification and new Patterns of Specialisation - Professionals and Experts in knowledge - intensive Business Services (KIBS), Working paper, Philipps-Universität Marburg, Alemania (2008).

Tarí, J.J. y M. García, Dimensiones de la gestión del conocimiento y de la gestión de la calidad: una revisión de la literatura.Investigaciones Europeas de Dirección y Economía de la Empresa: 15(3), 1135-2523 (2009).

Tunc, F., A. Beskese, y C. Kahraman, Prioritization of human capital measurement indicators using fuzzy AHP. Expert Systems with Applications, 1100-1112.(2007).

Yang, B. y H. Wang, Characterizing and Modeling the Structure of Competition Networks, Journal of Service Science and Management: 4(1), 8-14 (2011).

Yang, J., The knowledge management strategy and its effect on firm performance: A contingency analysis, Int. J.ProductionEconomics: 125, 215-223 (2010).

Zheng, W., B. Yang y G.N. McLeanc, Linking organizational culture, structure, strategy, and organizational effectiveness: Mediating role of knowledge management, Journal of Business Research: 63(7), 763-771 (2010). 